DOI: $10.4274 /$ jarem.galenos.2021.3915

J Acad Res Med 2021;11(1):69-74

\title{
The Risk Factors of Unplanned Hospital Readmission Following Percutaneous Nephrolithotomy
}

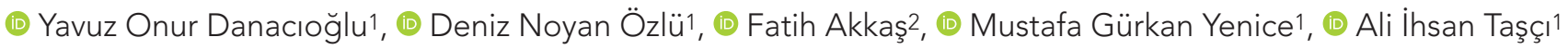 \\ ${ }^{1}$ University of Health Sciences Turkey, Bakırköy Dr. Sadi Konuk Training and Research Hospital, Clinic of Urology, İstanbul, Turkey \\ 2University of Health Sciences Turkey, Erzurum Regional Training and Research Hospital, Clinic of Urology, Erzurum, Turkey
}

Cite this article as: Danacıoğlu YO, Özlü DN, Akkaş F, Yenice MG, Taşçı Ail. The Risk Factors of Unplanned Hospital Readmission Following Percutaneous Nephrolithotomy. J Acad Res Med 2021;11(1):69-74

\begin{abstract}
Objective: Percutaneous nephrolithotomy (PNL) is currently recommended as the first treatment option for complex kidney stones larger than $2 \mathrm{~cm}$. Although it is a safe and effective treatment, emergency room admissions and hospitalisations occur because of PNL complications. This study aimed to examine patient-related independent risk factors that may cause unplanned hospital readmission after PNL.

Methods: Data of patients who were admitted to our clinic due to upper urinary system calculi and underwent conventional PNL operation between January 2015 and December 2019 were evaluated retrospectively. Hospital readmission was defined as unscheduled rehospitalisation within 30 days after discharge. The study cohort was divided into two groups: the readmission (group A) and non-readmission groups (group B). Post-operative complication scoring was performed according to the Clavien-Dindo classification. Multivariate logistic regression analysis was used to evaluate independent prognostic risk factors on readmission after PNL.

Results: A total of 390 patients were included in our study. Of patients, 24 (6.1\%) were readmitted to our clinic in the post-operative period. Gender, age, body mass index and stone volume were statistically similar between the groups. Based on multivariate analysis, the presence of Clavien $3 a$ and $3 \mathrm{~b}$ complications, post-operative blood transfusion, presence of preoperative hydronephrosis, American Society of Anaesthesiologist score and low stone density ( $<859$ Hounsfield units) were determined as significant independent risk factors for readmission.

Conclusion: Determination of preoperative risk factors will reduce hospital readmissions rates, thus reducing the potential burden on the health system and increasing patient comfort.

Keywords: Percutaneous nephrolithotomy, patient readmission, risk factors
\end{abstract}

\section{INTRODUCTION}

Percutaneous nephrolithotomy (PNL) is currently recommended as the first treatment option for complex kidney stones larger than $2 \mathrm{~cm}$ (1). It is also used for extracorporeal shockwave lithotripsy treatment-resistant lower pole stones bigger than $1 \mathrm{~cm}$ and patients with anatomical variation (2). Since the first use of PNL in 1976, a comprehensive modification has been performed to decrease post-operative complications, pain, hospital stay length and hospital readmission rate (3). In addition to technological improvements in endoscopic devices (flexible pyeloscope, flexible urethroscope, etc.), improvements in lithotripsy techniques increased the success of PNL by up to 90\% (4).

With its high success and low complication rates, PNL is regarded as a safe treatment method. Complications associated with PNL are generally related to perioperative neighbouring organ

ORCID IDs of the authors: Y.O.D. 0000-0002-3170-062X; D.N.Ö. 0000-0003-2435-5482; F.A. 0000-0002-4560-7426; M.G.Y. 0000-0002-5813-3565; A.I.T. 0000-0002-6943-6676.

Corresponding Author/Sorumlu Yazar: Deniz Noyan Özlü, E-mail: noyanozlu@hotmail.com
Received Date/Geliş Tarihi: 07.11.2020 Accepted Date/Kabul Tarihi: 24.02.2021

CC Copyright 2021 by University of Health Sciences Turkey, Gaziosmanpaşa Training and Research Hospital. Available on-line at www.jarem.org 
injuries (lung, pleura, colon, spleen, etc.), post-operative bleeding and urinary system infection (5). Because of unpredictable complications, emergency room (ER) admissions and unplanned hospital readmissions may occur in the post-operative period (6). In many countries, as an objective measure of the quality of health care, unplanned post-surgery readmission is considered. This situation causes both a decrease in the quality of life of patients and a severe financial burden on the healthcare system (7). More studies have been found in the literature on readmissions after urological surgeries and their causes in recent years $(8,9)$. These studies include general urological surgical procedures, and there are limited studies examining potential predictive factors for endourology and stone surgery $(10,11)$. This study aimed to examine patient-related independent risk factors that may increase the unplanned hospital readmission possibility after PNL.

\section{METHODS}

Data of 485 patients over the age of 18 years who were admitted to our clinic with upper urinary system calculi and underwent PNL operation between January 2015 and December 2019 were analysed retrospectively. The study protocol was approved by the local ethics committee of the Bakırköy Dr. Sadi Konuk Training and Research Hospital Ethics Committee, and the study was conducted according to the Declaration of Helsinki (approval number: 2020-17, approval date: 24.08.2020). Written informed consent, approved by our institutional review board, was obtained from all patients.

Hospital readmission was defined as unscheduled rehospitalisation within 30 days after discharge. Patients with a history of malignancy, concomitant surgery or readmitted to the hospital for other reasons were excluded from the study. Postoperative complication scoring was conducted according to the Clavien-Dindo classification (12).

Patients were evaluated using contrast-enhanced computed tomography (CT) and/or intravenous pyelography, which were taken before the operation. The preoperative total volume of stone $\left(\mathrm{cm}^{3}\right)$ was calculated from axial images with coronal reconstructions of non-contrast CT scan with length $\times$ height $\times$ width $\times \pi \times 1 / 6$ formula (13). Urine cultures of all patients were sterile preoperatively. Second-generation cephalosporin prophylaxis was given to all patients before the operation. All procedures were performed under general anaesthesia in the prone or supine position by three different surgeons with similar PNL experiences (Y.O.D., M.G.Y. and F.A.A.). Calculi were fragmented through pneumatic lithotripter (Vibrolith $\Theta_{1}$ Elmed, Ankara, Turkey) using $24 \mathrm{Fr}$ nephroscope (Karl Storz GmbH \& Co. KG, Tuttlingen, Germany) following ultrasonography-guided fluoroscopic access and extracted with the help of forceps. In all cases, a 14 Fr nephrostomy tube was placed in the renal pelvis or the involved calyx. If there were no contraindications, they were removed at the $24^{\text {th }}$ hour postoperatively. A routine ureteral catheter is not placed unless there are indications such as pelvic or ureter injury, high volume residual fragment or obstruction. In cases where they are required, ureteral catheters are removed 2-3 weeks later. Stone-free status of the patients was evaluated using direct urinary system graphy in the post-operative early period and by low dose non-contrast abdominal CT in the third month.

\section{Statistical Analysis}

Categorical data were presented as numbers and percentages. Data on continuous variables are presented as mean and standard deviation. Dependent t-test and Mann-Whitney U tests were used to compare the mean differences of normally and non-normally distributed data, respectively. Frequencies of categorical variables were compared using the Pearson chi-square test. A p-value of $<0.05$ was considered statistically significant. Logistic regression analysis was performed to determine readmission predictors after PNL. Multivariate logistic regression analysis was used to evaluate independent prognostic risk factors on readmission after PNL. Statistical analysis was performed using the Statistical Package of Social Sciences version 21 (IBM SPSS Statistics; IBM Corp., Armonk, USA).

\section{RESULTS}

A total of 390 patients were included in the study. The characteristics and demographic data of the patients are presented in Table 1. Of patients, 24 (6.1\%) were readmitted to our clinic in the post-operative period. The reasons for readmission after surgery were renal colic $(n=7,29.1 \%)$, urinary tract infection $(n=6,25 \%)$, haematuria $(n=6,25 \%)$, leakage $(n=2,8.3 \%)$, ureteral stent displacement $(n=1,4.1 \%)$, urinary retention $(n=1,4.1 \%)$ and urinothorax $(n=1,4.1 \%)$. The study cohort was divided into two groups: The readmission group (group $A ; n=24$ ) and nonreadmission group (group $B ; n=366$ ).

Group A consisted of 20 (83.3\%) male and 4 (16.7\%) female patients with a mean age of $44.8 \pm 17$ years and group B of 238 (65\%) male and 128 (35\%) female patients with a mean age of $44 \pm 13.8$ years. The mean stone volumes were $2,630 \pm 1,171$ and $2,311 \pm 1,036 \mathrm{~mm}^{3}$ in groups $A$ and $B$, respectively. No significant differences were noted between the two groups in gender, age, body mass index and stone volume ( $p>0.05)$. In the preoperative evaluation, the mean stone density was $710.7 \pm 451.2$ Hounsfield units $(H U)$ in group $A$ and $1004.8 \pm 324.7 \mathrm{HU}$ in group $B(p=0.001)$.

In all PNL procedures, 103 (26.4\%) were performed in the supine position and $287(73.6 \%)$ in the prone position. A total of $21(27.2 \%)$ and 56 (72.8\%) complications were observed in patients who underwent supine and prone PNL, respectively. In group $\mathrm{A}$, PNL was performed in 7 (29.1\%) and 17 (70.9\%) patients in the supine and prone positions, respectively. The positions in the procedures have no statistically significant difference in complication and rehospitalisation rates $(p=0.8$ and 0.752 , respectively). The rate of patients with Clavien $3 \mathrm{a}$ and $3 \mathrm{~b}$ complications was statistically higher in group $A$ than in group B ( $25 \%$ vs $3.3 \% ; p<0.001)$. In both groups, the American Society of Anaesthesiologists (ASA) score was significantly higher in group $A(p=0.047)$. The proportion of patients with ASA III score was $62.5 \%$ in group A and $6.6 \%$ in 
Table 1. Comparison of patients' characteristics according to readmission

\section{Variables}

Number of patients

\section{Sex, $n$ (\%)}

Male

Female

Mean age \pm SD (years)

Mean $\mathrm{BMI} \pm \mathrm{SD}\left(\mathrm{kg} / \mathrm{m}^{2}\right)$

Mean stone volume $\left(\mathrm{mm}^{3}\right)$

Mean stone density $\pm \mathrm{SD}(\mathrm{HU})$

Mean HD \pm SD (day)

Mean operation time \pm SD (min)

ASA score, $n$ (\%)

1

2

3

Post-operative complication, n (\%)

Clavien 0

Clavien 1

Clavien 2

Clavien $3 a$ and $3 b$

Post-operative blood transfusion, n (\%)

Positive

Negative

Preoperative hydronephrosis, $\mathrm{n}(\%)$

Positive

Negative

Residual stone, n (\%)

Positive

Negative

Staghorn calculi, $\mathbf{n}(\%)$

Positive

Negative

Preoperative culture positive, $\mathrm{n}(\%)$

Positive

Negative

Post-operative fever, $\mathrm{n}(\%)$

Positive

Negative

Preoperative nephrostomy catheter, n (\%)

Positive

Negative

Preoperative ureteral stent, n (\%)

Positive

Negative

Open stone surgery history, $\mathrm{n}(\%)$

Positive

Negative

Endourologic surgery history, $\mathrm{n}(\%)$

Positive

Negative

Surgical method, $\mathrm{n}(\%)$

Supine position

Prone position

\section{Group A (readmission)}

24

$20(83.3)$

4 (16.7)

$44.8 \pm 17$

$25.6 \pm 1.4$

$2,630 \pm 1,171$

$771.1 \pm 290.8$

$4.1 \pm 2.1$

$75.7 \pm 12.8$

$4(16.7)$

5 (20.8)

$15(62.5)$

$3(12.5)$

$5(20.8)$

$10(41.7)$

6 (25)

$14(58.3)$

$10(41.7)$

$15(62.5)$

9 (37.5)

$7(29.2)$

17 (70.8)

4 (16.7)

$20(83.3)$

6 (25.0)

$18(75.0)$

2 (8.3)

$22(91.7)$

2 (8.3)

$22(91.7)$

4 (16.7)

20 (91.7)

0 (0)

24 (100)

12 (50)

12 (50)

7 (29.2)

17 (70.8)
Group B (no readmission)

p-value

366

238 (65.0)

$128(35.0)$

$44 \pm 13.8$

$0.066^{\&}$

$26.8 \pm 3.2$

$0.802^{*}$

$2,311 \pm 1,036$

$0.076^{*}$

$0.148^{*}$

$1004.8 \pm 324.7$

$0.001^{*}$

$3.6 \pm 1.5$

$80.6 \pm 18.2$

$0.373^{*}$

$0.143^{\star *}$

$<0.001^{\&}$

136 (36.4)

214 (57.2)

24 (6.4)

ASA 3 vs ASA

1-2

$<0.001^{\&}$

$310(84.7)$

$34(9.3)$

$10(2.7)$

$<0.001$

$12(3.3)$

$20(5.5)$

$346(94.5)$

$<0.001^{\&}$

$132(36.1)$

234 (63.9)

$<0.001^{\&}$

76 (20.8)

$290(79.2)$

$0.330^{2}$

44 (12.0)

$322(88.0)$

$0.502^{\&}$

$20(5.6)$

338 (94.4)

$<0.001^{\&}$

50 (13.8)

$312(86.2)$

$0.446^{8}$

$18(5.0)$

344 (95.0)

$0.472^{2}$

50 (13.8)

312 (86.2)

$0.446^{8}$

48 (13.1)

318 (86.9)

$0.058^{2}$

$114(31.1)$

252 (68.9)

$0.056^{\&}$

$96(26.2)$

$270(73.8)$

\section{$0.752^{2}$}

SD: standard deviation, BMI: body mass index, HD: hospitalisation duration, HU: hounsfield unit, ASA: American Society of Anaesthesiologists, "Independent t-test, "Mann-Whitney U test, \&Pearson chi-square test 
the other group $(p<0.001)$. Post-operative blood transfusion was applied to $58.3 \%$ of patients in group $A$ and $5.5 \%$ of patients in group $B(p<0.001)$. Preoperative hydroureteronephrosis (HUN) was present in $62.5 \%$ and $36.1 \%$ of the patients in groups $A$ and $B$, respectively $(p<0.001)$. When the patients who received antibiotherapy due to positive preoperative urine culture were examined, there were statistically significant differences between the groups (group A: 25\%; group B: $5.6 \% ; p<0.001$ ).

According to multivariate logistic regression analysis, ASA score, presence of Clavien $3 \mathrm{a}$ and $3 \mathrm{~b}$ complications, post-operative blood transfusion requirement, presence of preoperative HUN, preoperative urine culture growth and presence of low stone density were determined as significant independent risk factors for readmission (Table 2). According to the receiver operating characteristic $(\mathrm{ROC})$ curve analysis, the threshold value for stone density was $859 \mathrm{HU}$, with a sensitivity of $66.7 \%$ and specificity of $65.7 \%$, and values under this threshold were regarded as low stone density. In predicting readmission, the area under the curve (AUC) values for preoperative HUN and low stone density ( $<859$ $\mathrm{HU}$ ) were 0.714 [95\% confidence interval (Cl) $0.557-0.872 ; \mathrm{p}=0.007$ ] and 0.768 (95\% $\mathrm{Cl} 0.677-0.858 ; \mathrm{p}=0.001)$, respectively (Figure 1 and Table 3).

\begin{tabular}{|c|c|c|c|}
\hline & OR & $95 \% \mathrm{Cl}$ & $p$-value \\
\hline \multicolumn{4}{|l|}{ Variables } \\
\hline ASA score & 1.990 & $0.866-4.573$ & $<0.001$ \\
\hline Clavien $3 a$ and $3 b$ complications & 2.998 & $1.864-4.789$ & 0.042 \\
\hline Post-operative blood transfusion & 2.450 & $0.594-10.112$ & $<0.001$ \\
\hline Preoperative hydronephrosis & 3.190 & 1.022-9.957 & 0.046 \\
\hline $\begin{array}{l}\text { Preoperative urine culture } \\
\text { positive }\end{array}$ & 1.120 & $1.100-1.160$ & 0.215 \\
\hline Hounsfield unit $<859$ & 3.669 & $1.176-11.449$ & 0.025 \\
\hline
\end{tabular}

\section{DISCUSSION}

The readmission rate of patients after PNL operation, which is frequently applied for treating kidney stones, and the predictive factors causing this were determined in our study. Studies in the literature provide us with an idea about the reasons and incidence of recurrence of general urological procedures. However, studies on readmission rates and predictive factors, especially in endourological stone surgeries, are limited in the literature. Unplanned hospital readmissions in the early post-operative period can also impair the quality of life of patients and the current functioning of urology clinics (14). Post-operative readmissions to the ER are negative indicators of the quality of healthcare services and cause significant economic burdens. In the United States of America, only in 2010 , insurance companies were paid $\$ 17.5$

\section{ROC Curve}

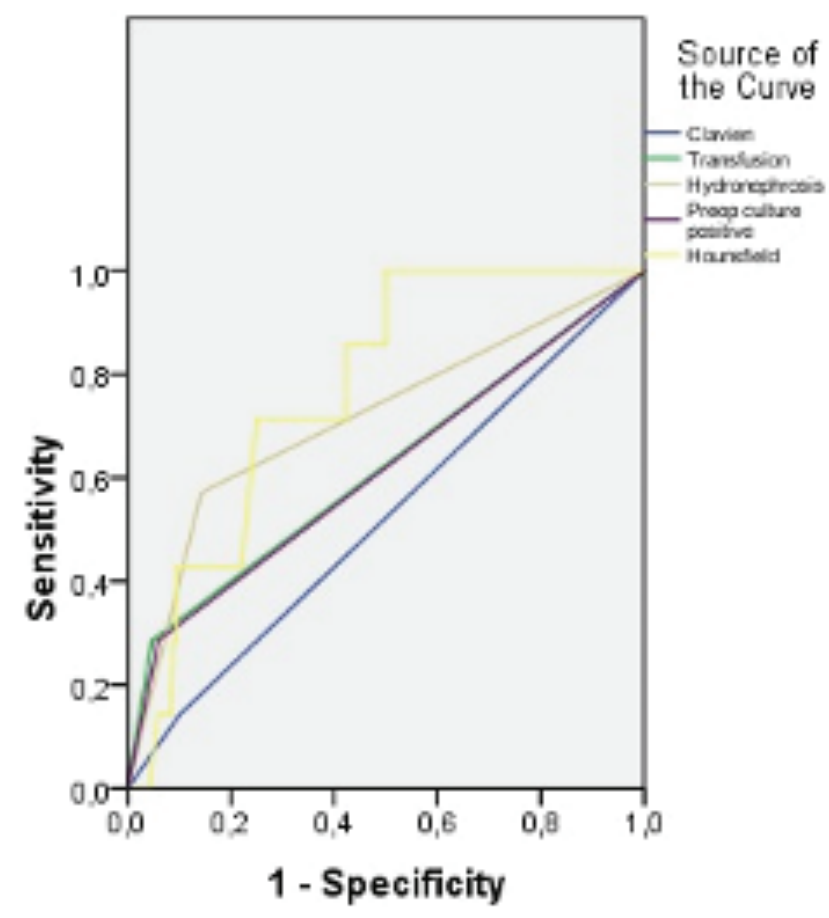

\section{Diagonal segments are produced by ties.}

Figure 1. Receiver operating characteristic curves of readmission predicting factors of patients ROC: receiver operating characteristic

Table 3. Receiver operating characteristic curve analysis for predictive factors for readmission

\begin{tabular}{|c|c|c|c|}
\hline & AUC & $95 \% \mathrm{Cl}$ & $p$-value \\
\hline \multicolumn{4}{|l|}{ Variables } \\
\hline Clavien $3 \mathrm{a}$ and $3 \mathrm{~b}$ complication & 0.521 & $0.362-0.680$ & 0.792 \\
\hline Post-operative blood transfusion & 0.619 & $0.447-0.791$ & 0.131 \\
\hline Preoperative hydronephrosis & 0.714 & $0.557-0.872$ & 0.007 \\
\hline $\begin{array}{l}\text { Preoperative urine culture } \\
\text { positive }\end{array}$ & 0.613 & $0.442-0.784$ & 0.152 \\
\hline Low Hounsfield score & 0.768 & $0.677-0.858$ & 0.001 \\
\hline
\end{tabular}

AUC: area under the curve, $\mathrm{Cl}$ : confidence interval, the cut-off value for $\mathrm{HU}$ was 859 , with a sensitivity of $66.7 \%$ and specificity of $65.7 \%$

billion for referrals to the hospital, and it was decided to impose sanctions on centres with high readmission rates (8). Unplanned hospital readmissions after surgery constitute a source of concern for all endourologists working in the field of stone surgery (5). When cystoscopy, transurethral bladder tumour resection, transurethral prostate resection, hydrocele excision and urethral sling surgeries, as five surgical procedures most applied in urology were examined in a study, the hospital readmission rate was $3.7 \%$. Cancer history, male gender, bleeding disorders and ASA score > III were detected as significant risk factors (8). In a similar study by 
Kumar et al. (14), male gender, smoking, chronic obstructive lung disease, diabetes mellitus (DM), coronary artery disease, bleeding disorders and ASA score $>$ III were risk factors for readmission. Consistent with the literature, a significantly higher readmission rate was detected in patients with an ASA score of III, and this was an independent predictive factor in our study. In the literature, the readmission rates after PNL were between $1.7 \%$ and $9 \%(6,14,15)$. In two different studies conducted in Turkey, readmission rates were $5.27 \%$ and $27.1 \%(10,11)$. In our study, the rate of hospital readmission was $6.5 \%$, which is consistent with the literature.

Advanced age was related to post-operative morbidity in urological surgeries (16). There is evidence in the literature that advanced age increases the readmission rate $(8,9,14)$. Johnston et al. (17) determined that the readmission rates after PNL were $18.7 \%$ and $7.1 \%$ in patients aged over 75 and under 18 years, respectively. Considering that our data exclude patients younger than 18 years, the mean age between both groups was statistically similar.

In endourological procedures such as $\mathrm{PNL}$, perioperative and post-operative complications related to urinary infections are common problems. The severity of these infections ranges from simple bacteriuria to severe sepsis, which can cause multiple organ failure (1). In a systematic review investigating PNL complications including 12,000 patients, fever and sepsis were observed at a rate of $10.1 \%$ and $0.5 \%$, respectively (18). The most common reason for hospital readmissions in the post-operative period is urosepsis (14). Risk factors for post-PNL sepsis include positive urine culture, stone size, infection stone, neurogenic bladder dysfunction, abnormal kidney anatomy, long operation duration, presence of nephrostomy tube and/or urethral catheter (19). In our current study, no significant difference was found between the two groups in terms of post-operative fever. Although a significant difference was observed among the two groups in preoperative urine culture positivity, it was not identified as an independent risk factor in multivariate analysis.

Perioperative and post-operative bleeding is a complication of PNL that may cause mortality. It can be seen in different stages of the operation such as percutaneous access, tract dilatation, stone fragmentation and post-operative period (5). The presence of DM, perioperative complications, operation duration, imaging method used during access (ultrasonic or fluoroscopic), tract dilatation technique, tract diameter and renal parenchymal thickness were significant risk factors for bleeding and the need for transfusion (20). In a multicentre prospective study, the blood transfusion rate after PNL was 5.7\% (21). In the study by Armitage et al. (6), readmission was observed because of haematuria or haemorrhage with a rate of $1.7 \%$ after PNL. Although haematuria and bleeding have not been examined separately, we concluded that blood transfusion, which may have a consequence, is an independent risk factor for hospital readmission.

Renal parenchymal thickness and stone density are factors that may affect PNL outcome (20). In the study examining complication rates, the operation time and blood loss were stated to increase in patients with a stone density of $<1000 \mathrm{HU}$ (22). Although data such as perioperative haemorrhage and decrease in haematocrit are indirectly related to readmission, there are no data in the literature examining the direct relationship between $\mathrm{HU}$ and readmission rates. Thus, this study is the first to determine HU as a risk factor for unplanned readmission within post-operative early period following PNL. In the ROC analysis, the threshold value for stone density was $859 \mathrm{HU}$, and a stone density lower than $859 \mathrm{HU}$ was a risk factor for patients' readmission. According to the ROC analysis, it was determined to have the highest AUC value. Although the composition of stones was not determined in the patients in our study, low HU determined in the readmission group constitutes the possibility of these patients having infection stones (1).

Complications due to residual stone fragments may be a marker for readmission (23). In the study in which a high stone burden was found to be a significant risk factor for readmission, it was concluded that this condition was associated with prolonged operation time and more residual stone fragments. It has been reported that if surgery is planned when patients are asymptomatic and the stone burden is not high, it may be possible to decrease the readmission rate (10). Although we did not find a significant difference between the two groups in terms of stone volume, the presence of preoperative HUN is an independent risk factor for readmission. Based on these data, surgeries performed with less stone burden in the early period and before HUN development may have a significant effect on readmission rates.

Complication rates reaching $83 \%$ were reported after PNL (5). In the study by Tepeler et al. (11), Clavien grades 1, 2, 3a+3b and 4 complication rates were $1.8 \%, 3.2 \%, 1.3 \%$ and $0.1 \%$, respectively. In our study, the post-operative complication rates in group $\mathrm{A}$ were significantly higher than in group B.

\section{Study Limitations}

One of the main limiting factors was the retrospective nature of our study. In addition, the comorbidities of the patients were not evaluated separately, and data on stone analysis, time until readmission and intraoperative complications were lacking in our study. The absence of stone localisation can be considered a limitation, but most of the patients who applied to our clinic have staghorn and semistaghorn stones. Thus, stone localisation of the patients could not be included in the study.

\section{CONCLUSION}

Hospital readmissions after PNL affect patient comfort and constitute a burden on the healthcare system. According to our study, ASA scores, the presence of Clavien 3a and 3b complications, the need for post-operative blood transfusion and the presence of preoperative HUN, preoperative urine culture growth and low stone density were risk factors for hospital readmissions. Choosing the patient and the surgical method according to these risk factors will reduce both the complication and readmission rates. Our study may help surgeons take constructive precautions 
in the treatment planning of patients with risk factors and may enlighten the way of future studies on this subject.

Ethics Committee Approval: The study protocol was approved by the local ethics committee of the Bakırköy Dr. Sadi Konuk Training and Research Hospital Ethics Committee, and the study was conducted according to the Declaration of Helsinki (approval number: 2020-17, approval date: 24.08.2020).

Informed Consent: Written informed consent, approved by our institutional review board, was obtained from all patients.

Peer-review: Externally peer-reviewed.

Author Contributions: Surgical and Medical Practices - Y.O.D., D.N.Ö., F.A., M.G.Y., A.I.T.; Concept - Y.O.D., F.A., A.I.T.; Design - M.G.Y., A.I.T., Y.O.D.; Data Collection and/or Processing - Y.O.D., D.N.Ö.; Analysis and/ or Interpretation - Y.O.D., F.A.; Literature Search - Y.O.D., D.N.Ö., M.G.Y.; Writing Manuscript - Y.O.D., D.N.Ö.

Conflict of Interest: The authors have no conflict of interest to declare.

Financial Disclosure: The authors declared that this study has received no financial support.

\section{REFERENCES}

1. Pradère B, Doizi S, Proietti S, Brachlow J, Traxer O. Evaluation of guidelines for surgical management of urolithiasis. J Urol 2018; 199: 1267-71.

2. Türk C, Petík A, Sarica K, Seitz C, Skolarikos A, Straub M, et al. EAU guidelines on interventional treatment for urolithiasis. Eur Urol 2016; 69 : 475-82.

3. Fernström I, Johansson B. Percutaneous pyelolithotomy: a new extraction technique. Scand J Urol Nephrol 1976; 10: 257-9.

4. Kallidonis P, Panagopoulos V, Kyriazis I, Liatsikos E. Complications of percutaneous nephrolithotomy: classification, management, and prevention. Curr Opin Urol 2016; 26: 88-94.

5. Basnet RB, Shrestha A, Shrestha PM, Joshi BR. Risk factors for postoperative complications after percutaneous nephrolithotomy. J Nepal Health Res Counc 2018; 16: 79-83.

6. Armitage JN, Withington J, van der Meulen J, Cromwell DA, Glass J, Finch WG, et al. Percutaneous nephrolithotomy in England: practice and outcomes described in the Hospital Episode Statistics database. BJU Int 2014: 113: 777-82

7. Squires D, Anderson C. U.S. health care from a global perspective: spending, use of services, prices, and health in 13 countries. Issue Brief (Commonw Fund) 2015; 15: 1-15.

8. Rambachan A, Matulewicz RS, Pilecki M, Kim JY, Kundu SD. Predictors of readmission following outpatient urological surgery. J Urol 2014; 192: 183-8.

9. Stone BV, Cohn MR, Donin NM, Schulster M, Wysock JS, Makarov DV, et al. Evaluation of unplanned hospital readmissions after major urologic inpatient surgery in the era of accountable care. Urology 2017: 109: 94100

10. Keskin SK, Danacioglu YO, Turan T, Atis RG, Canakci C, Caskurlu T, et al. Reasons for early readmission after percutaneous nephrolithotomy and retrograde intrarenal surgery. Wideochir Inne Tech Maloinwazyjne 2019; 14: 271-7.

11. Tepeler A, Karatag T, Tok A, Ozyuvali E, Buldu I, Kardas S, et al, Factors affecting hospital readmission and rehospitalization following percutaneous nephrolithotomy. World J Urol 2016; 34: 69-73.

12. de la Rosette JJ, Opondo D, Daels FP, Giusti G, Serrano A, Kandasami $\mathrm{SV}$, et al. Categorisation of complications and validation of the Clavien score for percutaneous nephrolithotomy. Eur Urol 2012; 62: 246-55.

13. Ito H, Kawahara T, Terao H, Ogawa T, Yao M, Kubota Y, et al. The most reliable preoperative assessment of renal stone burden as a predictor of stone-free status after flexible ureteroscopy with holmium laser lithotripsy: a single-center experience. Urology 2012; 80: 524-8.

14. Kumar M, Pandey S, Aggarwal A, Sharma D, Garg G, Agarwal S, et al. Unplanned 30-day readmission rates in patients undergoing endourological surgeries for upper urinary tract calculi. Investig Clin Urol 2018; 59: 321-7.

15. Beiko D, Elkoushy MA, Kokorovic A, Roberts G, Robb S, Andonian S. Ambulatory percutaneous nephrolithotomy: what is the rate of readmission? J Endourol 2015; 29: 410-4.

16. Sommer F, Ehsan A, Caspers HP, Klotz T, Engelmann U. Risk adjustment for evaluating the outcome of urological operative procedures. J Urol 2001; 166: 968-72.

17. Johnston AW, Jiang R, Alkazemi MH, Wolf S, Pomann G-M, Lipkin $M E$, et al. Nationwide readmission rates following percutaneous nephrolithotomy: does age matter? J Endourol 2019; 33: 704-11.

18. Seitz C, Desai M, Häcker A, Hakenberg OW, Liatsikos E, Nagele U, et al. Incidence, prevention, and management of complications following percutaneous nephrolitholapaxy. Eur Urol 2012; 61: 146-58.

19. Lojanapiwat B. Infective complication following percutaneous nephrolithotomy. Urol Sci 2016; 27: 8-12.

20. Kukreja R, Desai M, Patel S, Bapat S, Desai M. First prize: factors affecting blood loss during percutaneous nephrolithotomy: prospective study. J Endourol 2004; 18: 715-22.

21. de la Rosette J, Assimos D, Desai M, Gutierrez J, Lingeman J, Scarpa $\mathrm{R}$, et al. The clinical research office of the Endourological Society Percutaneous Nephrolithotomy Global Study: indications, complications, and outcomes in 5803 patients. J Endourol 2011; 25: 11-7.

22. Anastasiadis A, Onal B, Modi P, Turna B, Duvdevani M, Timoney A, et al. Impact of stone density on outcomes in percutaneous nephrolithotomy (PCNL): an analysis of the clinical research office of the endourological society (CROES) pcnl global study database. Scand J Urol 2013; 47: 50914.

23. Selby MG, Vrtiska TJ, Krambeck $A E$, McCollough $\mathrm{CH}$, Elsherbiny $\mathrm{HE}$, Bergstralh EJ, et al. Quantification of asymptomatic kidney stone burden by computed tomography for predicting future symptomatic stone events. Urology 2015; 85: 45-50. 